EDITORIAL

\title{
¿Cómo adaptamos la atención hospitalaria en pacientes con hiperglucemia y COVID-19?
}

\section{How do we adapt hospital care in patients with hyperglycemia and COVID-19?}

La pandemia por COVID-19 que atraviesa el mundo entero provocó una situación de crisis global en todos los sistemas sanitarios. Esta crisis nos obligó a realizar cambios emergentes para lograr una adecuada atención de los pacientes hospitalizados en áreas críticas y no críticas. Por lo cual, el personal de salud (PS) enfrentó nuevos desafíos, y recurrió a herramientas de uso ambulatorio (como el monitoreo continuo de glucosa y la telemedicina, que facilitaron espacios de comunicación del PS con el paciente hospitalizado), y también utilizó la tecnología para la transmisión de diagnósticos y tratamientos para pacientes a distancia.

Desde el inicio de la pandemia se describió a la diabetes mellitus (DM) como un factor de riesgo de mayor morbilidad y mortalidad por COVID-191. Rápidamente se conocieron y comunicaron los diversos mecanismos acerca de cómo el virus SARS-CoV-2 provoca hiperglucemia, tanto aguda como crónica, en ocasiones de novo y en otros casos descompensado metabólicamente a pacientes con DM preexistente ${ }^{2}$. A su vez, el estado de hiperglucemia favorece la replicación y diseminación del virus, y agrava la COVID-19. Esto provocó que las unidades de cuidados intensivos (UCl) se poblaran de pacientes COVID-19 con hiperglucemia, primaria o secundaria al virus o al uso de glucocorticoides, como parte del tratamiento de la neumonía por COVID-19. Esta nueva realidad ocasionó escasez de suministros, falta de equipos de protección personal para los trabajadores de la salud, déficit de insumos y una carga sobre el sistema de salud en general.

El desafío fue evaluar cómo abordar el control glucémico en los pacientes con COVID-19 hospitalizados para mejorar y reducir las complicaciones hospitalarias y la duración del tiempo de internación. Y además los nuevos objetivos son atenuar el riesgo y tiempo de exposición del PS, disminuir el riesgo de contagios y evitar la escasez de los equipos de protección de los profesionales (EPP). Los pacientes tratados con infusión de insulina endovenosa (IIEV) en áreas críticas requieren habitualmente un control de glucemia capilar horario o cada 2 horas, mientras que aquellos insulinizados que se encuentran en áreas no críticas, necesitan cuatro a cinco controles por día. En ambos casos, al tratarse de pacientes COVID-19, aumenta la frecuencia de contac- to del PS, lo que incrementa el riesgo de contagio, y la carga en tiempo y costos que genera un frecuente cambio de los EPP.

Para alcanzar aquellas viejas y nuevas metas, se recurrió al uso de tecnologías más conocidas y validadas en el paciente ambulatorio como el monitoreo continuo de glucosa (continuous glucose monitoring, CGM), pero en este caso aplicado a pacientes hospitalizados. A comienzos de 2020, los dispositivos de CGM en forma emergente y transitoria se aprobaron para pacientes hospitalizados con COVID-19. Rápidamente se desarrollaron ensayos clínicos pilotos que estudiaron su eficacia y seguridad en pacientes adultos y pediátricos, hospitalizados tanto en áreas críticas como en no críticas, con resultados positivos que afirmaban una viabilidad de los CGM para alcanzar los objetivos clínicos y de protección al de PS ${ }^{3,4}$. En Argentina, médicos e ingenieros en forma conjunta, realizaron los primeros ensayos clínicos en pacientes adultos y pediátricos, hiperglucémicos en estado crítico a quienes se ajustó la IIEV según valores y tendencia de la glucosa mediante CGM. Estos datos podían visualizarse en los teléfonos celulares del PS o en una computadora mediante una aplicación desarrollada en Argentina por ingenieros del CONICET5. La modificación de la velocidad de la IIEV solo se ejecutaba cuando el valor de glucosa estaba por fuera de los rangos objetivos (140 a $180 \mathrm{mg} / \mathrm{dll}$ ) y según su tendencia, lo cual redujo el número de contactos del PS con el paciente y adicionalmente se alcanzó un mejor control metabólico. La descripción detallada sobre cómo se implementó y los resultados de dicha experiencia argentina en la población pediátrica se publican en el presente número ${ }^{6}$.

Otros estudios similares en pacientes COVID-19, hospitalizados en áreas no críticas con insulinoterapia subcutánea (ISC), fueron entrenados para el uso CGM y desde la habitación comunicaban telefónicamente sus valores de glucosa y tendencias al PS, o bien el PS podía visualizar la glucosa en tiempo real y continuo a través de aplicaciones o software de cada dispositivo tecnológico (FreeStyle LibreView, Carelink-Medtronic, Dexcom). Los resultados fueron concordantes: redujeron la carga de atención, el número de contactos y riesgo de contagio del PS, y se agregó un menor riesgo de 
hipoglucemias al considerar la tendencia a la glucosa en la decisión sobre los cambios de dosis de insulina7,8.

Sin embargo, la planificación del uso del CGM en instituciones hospitalarias es una tarea compleja que requiere de una transformación continua en la atención del paciente diabético y que compromete múltiples áreas ${ }^{9,10,11}$ :

- Área clínica: comprende al personal médico y enfermería que asiste a pacientes con DM en forma general o como equipo especializado y deberían definir: a) criterios para incluir a los mejores candidatos a emplear CGM en $\mathrm{UCl}$ o en sala general, por ejemplo, la indicación por ahora más consensuada es el uso de CGM en pacientes hiperglucémicos con COVID-19 u otra enfermedad infecciosa de alta contagiosidad en tratamiento con IIEV. Al evitar el control horario de la glucemia capilar, se reduce el riesgo de contagio, el tiempo y costo del equipamiento del PS para hacer cada control, con el agregado que el dato de tendencia de glucosa disminuye el riesgo de hipoglucemias; b) criterios para no recomendar el CGM, por ejemplo, aquellos pacientes con hipo o hiperglucemia grave (es decir, glucemia de $<40 \mathrm{mg} / \mathrm{dL}$ o $>500 \mathrm{mg} / \mathrm{dL}$ ), o en anasarca, shock hipovolémico o con infecciones en el sitio de punción del sensor; c) metodología y personal responsable para proporcionar herramientas educativas para formar adecuadamente a pacientes, enfermeras y personal médico tratante una vez que el paciente tenga la indicación del CGM; d) responsables y método para establecer el control de calidad de los dispositivos empleados en el CGM.

- Área informática: mediante los tecnólogos de informática hospitalaria, médicos e ingenieros se definirán las plataformas de recolección y almacenamientos de los datos provenientes del CGM, y la manera para que el PS pueda visualizar los valores de glucosa

\section{BIBLIOGRAFÍA}

1. Zhou F, Yu T, Du R, Fan G, Liu Y, Liu Z, Xiang J, Wang Y, Song $B, G u X$. Clinical course and risk factors for mortality of adult inpatients with COVID-19 in Wuhan, China: a retrospective cohort study. Lancet 2020; 395:1054-1062.

2. Muniyappa R, Gubbi S. COVID-19 pandemic, coronaviruses, diabetes mellitus. NHI, Bethesda, Maryland. Am J Physiol Endocrinol Metab 2020; 318(5):E736-E741.

3. Ushigome $E$, Yamazaki $M$, Hamaguchi $M$, et al. Usefulness and safety of remote continuous glucose monitoring for a severe COVID-19 patient with diabetes. Diabetes Technol Ther 2020; 22:1-3.

4. Agarwal S, Mathew J, Davis G, Umpierrez G, et al. Continuous glucose monitoring in the intensive care unit during the $\mathrm{CO}$ VID-19 pandemic. Diabetes Care 2021 Mar; 44(3):847-849.

5. Garelli F, Sánchez-Peña R, Krochik G, Grosembacher L, et al. Remote glucose monitoring platform for multiple simultaneous patients at coronavirus disease 2019 intensive care units: case report including adults and children. Diabetes Technology \& Therapeutics 2021; Vol 23, Num 5. DOI:10.1089/dia.2020.0556 retrospectivamente y en tiempo real, en lo posible a través de la historia clínica informatizada ${ }^{10,11}$

- Área institucional: el hospital debería asegurar la provisión de los componentes necesarios para el CGM, coordinando con los laboratorios su provisión y la metodología a emplear para el control de calidad, además de crear un entorno institucional que facilite el uso del CGM, y encuestar al PS y pacientes para evaluar resultados y satisfacción.

La adaptación a la virtualidad que nos impuso la pandemia migra gradualmente del entorno ambulatorio al ámbito hospitalario. El uso de tecnología como método para acceder a consultas o datos de nuestros pacientes en forma remota hizo posibles diagnósticos y tratamientos también en el paciente hospitalizado. En el caso del uso de los CGM en los pacientes con COVID-19 e hiperglucémicos, además de contribuir con favorables resultados clínicos, se lograron beneficios muy importantes en la protección de contagios, disminución de la carga en tiempo y costos asociados a la reducción en el uso del equipamiento de protección en el PS. No obstante, recién estamos en el inicio de una prolongada tarea que nos incluye a todos los actores institucionales y que requiere aún de más investigaciones que evalúen eficacia y seguridad de los CGM en diversas situaciones clínicas o quirúrgicas del ámbito hospitalario para asegurar su validación en forma definitiva. Lo importantes es ser protagonistas y participar en esta nueva era tecnológica aplicada a la salud.

Dr. Luis A. Grosembacher Jefe de la Sección Diabetes Servicio de Endocrinología y Metabolismo Hospital Italiano de Buenos Aires

6. Krochik G, Prieto M, Martínez C, et al. Reporte de altos requerimientos de insulina en pacientes críticos pediátricos con COVID-19. Experiencia con monitoreo remoto continuo de glucosa. Revista de la Sociedad Argentina de Diabetes 2021; Vol. 55 N $^{\circ}$ : 70-74.

7. Reutrakul S, Genco M, Salinas H, et al. Feasibility of inpatient continuous glucose monitoring during the COVID-19 pandemic: early experience. Diabetes Care 2020; 43: e137-e138.

8. Shehav-Zaltzman G, Segal G, Konvalina N, Tirosh A. Remote glucose monitoring of hospitalized, quarantined patients with diabetes and COVID-19. Diabetes Care 2020; 43:e75-e76 4.

9. Galindo R, Aleppo A, Klonoff D, et al. Implementation of continuous glucose monitoring in the hospital: emergent considerations for remote glucose monitoring during the COVID-19 pandemic. Journal of Diabetes Science and Technology 2020; 14(4):822-832.

10. Galindo R, Umpierrez G, Rushakoff R, et al. Continuous glucose monitors and automated insulin dosing systems in the Hospital Consensus Guideline. Journal of Diabetes Science and Technology 2020; 14(6):1035-1064.

11. Pérez-Guzmán M, Trisha-Shang T, Zhang J, et al. Continuous glucose monitoring in the hospital. Endocrinol Metab 2021; 36:240-255. 